\title{
P53: Its Role in Carcinogenesis And Also A Novel Target for Anti-Cancer Therapy
}

\author{
Dr.Sujan Narayan Agrawal \\ Department Of Surgery. Late BRKM Government Medical College, Jagdalpur (Bastar). Chattisgarh. \\ Aayush And Health Science University, Raipur Chattisgarh,India
}

\begin{abstract}
: $p 53$ is a nuclear binding phosphoprotein, which regulates the gene expressions and controls several key genes involved in gene regulation. It facilitates DNA repair. If the damage is beyond repair, p53 triggers apoptosis of the cell. p53 has been shown to regulate apoptosis in both transcription dependent and independent manner.

Various types of genotoxic and non genotoxic stresses can lead to p53 activation like radiations, mutagens like Aflotoxins, benzopyrines, alkylating agents etc. and also the agents which damage mitotic spindle, cause ribonucleotide depletion, hypoxia, heat stroke, exposure to nitric acid etc. These damages are either repaired or apoptosis is triggered.

p53 pathways play a critical role in prevention of carcinogenesis. It may turn oncogenic when there is loss of function, dominant negative activity and oncogenic activity of mutant $p 53$. It plays a critical role in oncogenesis by several mechanisms, so targeting p53 is a favorite modality for developing a new approach in cancer therapeutics. The approach includes gene therapy to restore 553 function, inhibition of p53-Mdm interaction, restoration of mutant p53 to wild p53, targeting p53 family proteins, eliminating mutant p53 and p53 based vaccine.

It is very interesting to study p53 molecule in this perspective because, this unique molecule, has the property to trigger oncogenesis as well as it can be targeted therapeutically to treat cancers.
\end{abstract}

Keywords: p53, gene therapy, ONYX-015, p53-Mdm-2, p53 based vaccine.

\section{Introduction}

p53 is a nuclear DNA binding phosphoprotein that normally exists as homotetramer or complex of tetramers. It is transcriptional activator of a specific set of target genes, and can exert transcriptional repression probably by interaction with transcription factors or the general transcription machinery. It interacts directly with cellular proteins. Loss of p53 function can contribute to genomic instability within cells. p53 is important in preventing cancers because of its unique functional capabilities. It regulates gene expression and control several key genes involved in growth regulation. It facilitates DNA repair. If the damage is beyond repair, p53 triggers apoptosis of these cells, besides DNA damages, p53 dependent apoptosis is also induced by inappropriate oncogene activation, certain cytokines, hypoxia, heat shock, telomerase erosion etc. DNA damage can be due to exposure to radiation or drugs, causing damage to signals, the activation of cellular checkpoint kinase, such as ATM (Ataxia telangiactesia mutated) and ATR (ATM and Rad-3 related), which then leads to phosphorylation of p53. p53 is normally sequestrated by Mdm2. Phosphorylation of p53 disrupts p53's interaction with $\mathrm{Mdm} 2$ and ushers its activation. [1].

p53 then holds the cell at check point until the damage is repaired. If the damage is irreversible, apoptosis is triggered. Oncogene such as myc, Ras V12 and E2F-1 have also been shown to induce apoptosis by indirectly activating p53 via activation of ARF (Alternating Radiation Frame). ARF acts by sequestering Mdm2 thus releasing p53. [2].

P53 has been shown to regulate apoptosis in both, a transcription dependent and independent manner. In the transcription dependent pathway p53 activates the expression of several proapoptotic proteins such as PUMA, Bax and BID, which is involved in the regulation of the intrinsic cell death pathway as well as upgrade CD95 (Fas/Apo1) and DR5 receptors which mediate the extrinsic cell death signals. In addition, transcriptional activation of proapoptotic protein $\mathrm{p} 53$ has also been shown to suppress anti-apoptotic proteins such as surviving. [3].

\section{P53 Structure And Function}

The human TP53 gene spans $20 \mathrm{~kb}$ on chromosome band 17p13.1. The gene is composed of 11 exons, the first of which is noncoding. Its promoter does not contain TATA box but harbours a number of consensus binding sites. For common, transcription factors such as Spl, NF-kappaB or C-Jun. Despite these potential sites for transcriptional regulation, the expression of TP53 is constitutive and ubiquitous, most of the protein regulation taking place at the post translational level. 
The p53 protein is a nuclear phosphoprotein, composed of 393 amino acids in human. It has five structural and functional domains i.e. an N-terminal transactivation domain, a protein rich regulatory domain, an oligomerization domain and a C-terminal domain involved in the regulation of DNA binding. The most common mutation that occurs in cancer alters this structure either by abrogating protein-DNA contacts or by disrupting protein folding.

Various types of genotoxic and non genotoxic stresses can lead to p53 activation, like;-

$>$ Gamma or UV radiation, free radical damage, inhibition of topiosomarase, which causes single or double strand break in DNA.

$>$ Mutagens that form bulky DNA adducts like Aflotoxins, benzopyrines, alkylating agents, etc.

$>$ Agents that block elongation by RNA polymerization.

$>$ Agents that cause damage to mitotic spindle, ribonucleotide depletion, hypoxia, heat stroke, exposure to nitric acid etc.

$>$ Once activated, p53 can trigger several cellular events, via two distinct parallel pathways i.e. transcription dependent and/or transcription independent pathways. However the response of p53 activation e.g. cell cycle arrest or apoptosis depends upon the nature and amplitude of inducing signals and also the tissue and cell type. It is also important to understand that the two biological responses may co-exist within the same tissue. [4]

$>$ Its role in carcinogenesis: p53 and its downstream pathways play a critical role in preventing tumour formation. Its role in oncogenesis may be described under three main actions i.e.

1. Loss of function.

2. Dominant negative activity of mutant $\mathrm{p} 53$.

3. Oncogenic properties of mutant $\mathrm{p} 53$

\subsection{Loss of function}

More than $50 \%$ of cancer patients' harbour somatic mutation on p53 gene and about $80 \%$ mutations are missense mutations. The germ line p53 mutation causes a rare type of cancer predisposition disorder called LiFraumeni syndrome (LFS). Both somatic and germ line mutations are usually followed by loss of heterozygosity $(\mathrm{LOH})$ during tumour progression, which results in the inactivation of the remaining wild type alleles of p53. So the loss of function of p53 causes genomic instability, metastasis, resistance to chemotherapy and radiotherapy, poor patient survival and tumour progression.

\subsection{Dominant negative activity of mutant p53.}

The mutated p53 is over expressed in human tumours. This mutant p53 not only loses its tumour suppressive function but also has dominant negative activity on remaining wild type p53. It leads to accelerated tumour development and its growth. [5]

\subsection{Oncogenic properly of mutant p53.}

Mutant p53 acquires oncogenic properties that lead to "gain-of-function". Several mechanisms are attributed to this "gain-of-function" phenomenon. e.g.

2.3.1. Mutant $\mathrm{p} 53$ can inhibit the function of the $\mathrm{p} 53$ family proteins i.e. $\mathrm{p} 63$ and $\mathrm{p} 73$ by protein-to-protein interaction. It is found that mutant p53 inhibits p73 and p63 only when it is present in excess. (of p63, and p73). This situation is common in tumours. [6].

2.3.2. Regulation of gene transcription by mutant $\mathrm{p} 53$ is an important "gain-of-function" mechanism. Mutant p53 have the ability to activate the transcription of multi drug resistance 1 (MDR1) gene, which causes drug resistance in mutant p53 expressing cancer cells. Besides MDR1 mutant, p53 has been implicated in the transcriptional regulation of several genes including PCNA, $c-m y c$, FAS, bcl-x1 and VEGF. The transcriptional regulation of these specific genes by mutant p53 may be modulated through preferential binding to structural DNA motif such as non-B DNA structures. [7]. It can do so, through interaction of mutant p53 with sequencespecific transcription factor.

2.3.3. Mutant $\mathrm{p} 53$ inhibits the DNA repair pathway and thus have gain-of-function and genetic instability. [8] 


\section{Targeting P53 Signalling For Cancer Therapy: The Approaches}

The p53 plays a critical role in tumorogenesis by several mechanisms. So targeting p53, is a favourite target and a novel modality for developing a new approach to cancer therapeutics. These approaches include;-

3.1. Gene therapy to restore p53 function.

3.2. Inhibition of $\mathrm{p} 53-\mathrm{Mdm} 2$ interaction.

3.3. Restoration of mutant p53 to wild type p53.

3.4. Targeting the $\mathrm{p} 53$ family proteins.

3.5. Eliminating mutant $\mathrm{p} 53$.

3.6. 553 based vaccine.

\subsection{Gene therapy to restore p53 function}

Delivery of wild type p53 by virus in to the cancer cells is a direct strategy to rescue p53 activity in cancers. It is believed that restoration of p53 function in tumour cells may block tumour development and may sensitize cells to cytotoxic killing thus improving therapeutic response.

\subsubsection{Retrovirus mediated p53 gene therapy}

Retrovirus is an attractive candidate for cancer gene therapy application because they integrate in a stable form into the genome of infected cells and they require cell division for transduction. Retrovirus has been used in the majority of approved gene transfer clinical protocols. A phase 1 clinical trial was undertaken to determine the toxicity of retrovirus mediated delivery of wild type p53. [9].

Nine patients with non-small cell lung cancer for whom conventional treatment failed, were included in the study. The result shows no significant vector related toxic side effects up to five months after treatment. Apoptosis was more frequent in post treatment biopsies than pre-treatment biopsies. Retrovirus based gene therapy has three major drawbacks i.e.

$>$ They may cause damage to genome.

$>$ They may produce high titre of virus.

$>$ Their efficacy for transduction is relatively low especially in non-replicating cells.

\subsubsection{Adenovirus mediated $\mathrm{p} 53$ gene therapy}

The use of adenovirus is another favourite strategy to TP53 gene replacement therapy. It has several advantages over retrovirus. Their activity is not limited to actively proliferating cells. It has low toxicity profile. They do not integrate into the genome, so there is no risk of insertional mutagenesis.

In vivo and in vitro studies have demonstrated that adenovirus delivery of wild type p53 (Ad-p53) triggers a dramatic apoptosis and tumour regression response in various cancer types including head and neck cancers, lung cancers, prostate cancers, cervical cancer, ovarian cancer and gliomas etc.

The Ad5 CMV-p53 (Advexin ${ }^{\circledR}$, Gendicine ${ }^{\circledR}$ ) has been tested in phase I to phase IV clinical trials for the treatment of patients with recurrent malignant gliomas, head and neck cancers, hepatocellular carcinoma, nasopharyngeal carcinoma and ovarian cancers. The complete response rate, five year survival rate and five year disease free survival rates were significantly increased in the group receiving Ad-p53, combined with radiotherapy, as compared to group receiving radiotherapy alone. [10]. The limitation to this approach is that the virus is not able to infect every cancer cell due to limitation of virus delivery and that host antibodies reduce adenovirus infectivity.

ONYX-015 is an oncolytic antivirus therapy. It selectively replicate, in p53 dysfunctional tumour cells and results in, the specific lysis of those cells. Phase-I clinical trials have shown its efficacy with non-significant side effects. [11]. Its approach is essentially different from 'replacement' gene therapy. Indeed it does not involve transfer of a TP53 gene into the cell but requires the introduction of genetically modified (E1Bdefective Adenovirus) virus that will take advantage of dysfunctional p53 in cancer cells to selectively kill them.

\subsection{Inhibition Of The P53-Mdm2 Interaction}

Mdm2 is an E3 ubiquitin ligase which regulates p53 by feedback mechanism. Mdm2 is up regulated by p53; it interacts with $\mathrm{p} 53$ to promote polyubiquitination and subsequent proteosome dependent degradation of p53. Mdm2 is frequently amplified in human cancer. [12]. Due to critical inhibitory effect of Mdm2 on p53, targeting the interaction of Mdm2 with p53 is a potential therapeutic strategy. Some promising inhibitors p53Mdm2 is as below:-

3.2.1. Nutlin-3:- It is a potent and selective inhibitor of Mdm2-p53 interaction. It binds to Mdm2 mimicking the crucial amino acid residue of p53 that are essential for Mdm2 binding. It has been shown to block cells 
in $\mathrm{G} 1$ and $\mathrm{G} 2$ phases and induce apoptosis in a wild-type p53 dependent manner.

Treatment by Nutlin-

3a shows effective tumour growth inhibition and tumour shrinkage at non-toxic doses in human cancer

3.2.2. RITA: - It is a small molecule compound and is found to prevent the interaction of Mdm2 with p53. In contrast to Nutlin which binds to Mdm2, RITA binds to $\mathrm{p} 53$ thereby preventing Mdm2 interaction. It induces p53 dependent growth inhibition and apoptosis in tumour cells. [14].

3.2.3. MI-219:- It is a second class of Mdm2 inhibitors. The discovery of MI- 219 has demonstrated the utility of crystal structure guided drug design. It blocks the interaction of $\mathrm{p} 53$ with mdm2 by mimicking critical residue of the $\mathrm{p} 53-\mathrm{mdm} 2$ complex interface. It activates the $\mathrm{p} 53$ pathway and triggers apoptosis in $\mathrm{p} 53$ wild type cancer cells, but not in p53 mutant cancer cells. In a mouse model with wild type p53 human cancer xenograft, MI-219 induces tumour regression with little toxicity to normal tissue. [15].

3.2.4. RG-7112:- It is the first Mdm2 inhibitor which was developed in clinical trials by Hoffman-LA Roche, USA. It is a member of Nutlin family with improved potency and pharmacological properties. It activates the p53 pathway leading to cell cycle arrest and apoptosis in wild type p53 expressing cells. Preliminary clinical data indicates that RG7112 is well tolerated in patients and provides initial evidence that clinical activity is consistent with targeting the Mdm2-p53 interaction. [16].

The key limitation of inhibitors of $\mathrm{p} 53-\mathrm{Mdm} 2$ interaction is that they are only effective in wild type p53 expressing cancer cells, and they have no effect on mutant p53 expressing cancer cells. Wild type of p53 may be over expressed in the normal cells, thus it may lead to toxicity to normal cells. The system which show high proliferative index like haemopoietic system, intestinal epithelium may show toxicity to this agent due to cell cycle arrest and apoptosis.

\subsection{Restoration Of Mutant Type P53 To Wild Type P53.}

Mutant p53 is specifically over expressed in tumour cells, representing a tumour specific target. The restoration of the over expressed mutant p53 to wild type p53 could result in massive apoptosis in tumour cells. The rescue strategy depends upon mutant type p53 to wild type p53.

3.1 In tumour harbouring a DNA contact mutant, the attempts were to introduce the functional group, that create new contacts or to stabilise the scaffolding positioning in remaining contact sites. [17]. 3.2. The function of confirmation mutants can be restored by specific small peptides or small

molecules that aid the proper folding of the unfolded p53 conformation. [18].

3.3 PRIMA-1 and MIRA-1:- p53 reactivation and induction of massive apoptosis (PRIMA-1), and mutant p53 reactivation and induction of rapid apoptosis (MIRA-1) are two classes of compounds that suppress the growth of cancer cells expressing the exogenous mutant p53. They restore the sequence specific DNA binding and change the mutant p53 conformation to wild type, leading to transactivation of p53 target gene. [19]. both compounds showed antitumor activity in multiple cancer cell lines without apparent normal tissue toxicity. The PRIMA-1/PRIMA-1 (mct) has also shown to sensitize the lung cancer cells to action of Adriamycin and radiation to prostate cancer cells.

3.4. Phikan 083:- It is a carbazole derivative. It was identified by in silico analysis of the crystal structure of p53 Y220C mutant protein using virtual screening and rational drug design. It can selectively bind to a unique pocket in $\mathrm{p} 53$ Y220C mutant. [20].

3.5. Ellipticine: - It is a natural compound derived from Aspidos perma williansil anticancer and cytotoxic are well known in various tumours. It is shown to change the p53 conformation from mutant to wild type, restored the sequence-specific DNA binding and transactivation of p53 driven luciferase reporter, and activated mutant p53 to induce p53 target genes, p21 and mdm2 in mouse xenograft tumour tissues. [21]. However the toxic side effects have limited EPC to be developed into an anticancer therapeutic agent.

3.6. WR-1065:- WR-1065 (aminothiol) is an active metabolite of the cytoprotector amifostine and acts as a classic scavenger of reactive oxygen species. It was found to activate both wild and mutant p53 and increase the expression of $\mathrm{p} 53$ target genes in a manner, independent of DNA damage. [22].

3.7. NSC 319726:- It was identified by in silico analysis with the NC160 cell-line penal. The screening was designed to identify the compounds with increased sensitivity in a penal of cell lines carrying p53 mutations relative to wild type p53 tumour cells. NC 319726 was found to restore wild type p53 activity in R175H mutant cancer cell lines. [23].

3.8. p53R3:- It is a recently identified small molecule compound. It was found to enhance the recruitment of both wild and mutant $\mathrm{p} 53$ to target promoters and to induce the expression of a number of p53 target genes. Biologically, the compound induces mutant $\mathrm{p} 53$ dependent growth arrest and sensitises TRAILinduced cell death in multiple glioma cell lines. [24]. 
3.9. CP-31398:- Cp- 31398 was the first small molecule that has activity to change p53 confirmation from mutant to wild type. It was found to stabilize the core domain and enhance transcriptional activity of p53 in living cells. It had no effect on p53-mdm2 binding, did not caused DNA damage response, or induce p53 phosphorylation at ser 15 or 20, but rather it increased wild p53 levels by reducing p53 ubiquitination. [25].

3.10. SCH 529074:- It targets the mutant $\mathrm{p} 53$. It binds to the DNA binding domain of mutant $\mathrm{p} 53$, stabilizes mutant p53 and induces p53- dependent apoptosis. [26].

\section{Targeting P53 Family Proteins.}

P53 has two more family members, p73 and p63 with significant sequence homology among them. Like p53 they also bind to the p53 specific DNA binding motif, transactivate p53 downstream target genes, and suppress tumour cell growth by inducing growth arrest and apoptosis. [27].

Unlike p53, p73 and p63 is rarely mutated in human cancers but could be inactivated by binding to a subset of p53 mutants. Targeting p73 and p63 has been proposed for cancer therapy with approaches including gene therapy, small peptides and small molecules. [28].

\section{Eliminating The Mutant P53}

Mutant p53 acquires dominant negative activity and oncogenic function, and is, highly expressed in majority of human tumours. Thus targeting mutant p53 can be explored as a therapeutic strategy to manage tumours that depend upon mutant $\mathrm{p} 53$ for their survival.

5.1. 17AAG:- small molecules that degrade mutant p53 are promising chemicals in anti-cancer therapy. The molecular chaperon, HSP90 can interact mutant p53, consequently stabilizing the mutant p53 confirmation. The inhibitors of HSP90, 17AAg decrease mutant p53 by destroying the complex of mutant $\mathrm{p} 53$ for its degradation. [29].

5.2. SAHA: - Histone deacetylase HDAC6 is a positive regulator of HSP90 chaperone activity by regulating HSP90 deacetylation. Histone deacetylase (HDAC) inhibitor SAHA exhibits preferential cytotoxicity for mutant $\mathrm{p} 53$ human cancer cells, due to its ability to degrade mutant $\mathrm{p} 53$ by inhibition of the HDAC 6HSP90 chaperone axis. [30].

\section{P53 Based Vaccine.}

Human cancer shows a high titre of mutant p53 in their cancer cells; the wild type p53 protein has a very short half life and is therefore present in very low levels in normal cells. This different level of p53 expression between normal and cancer cells provides a basis for p53 immuno-therapy. The antibodies against p53 have been found in patients with various types of cancers, which indicate that the human immune system can recognise and respond to tumour associated $\mathrm{p} 53$.

6.1. p53-SLP:- p53-SLP (P53-synthetic lung peptide) has undergone phase I/II clinical trial for treatment of metastatic colorectal cancer. It is found to be non- toxic and p53 specific T-cell responses were induced in 9 out of 10 colorectal patients. [31].

6.2. INGN-225:- In a phase I/II clinical trial of INGN-225, a p53 modified adenovirus-transduced dendritic cell vaccine for the treatment of small cell lung cancer (SCLC) was reported. INGN-225 was well tolerated and a specific anti-p53 immune response was observed in 18 out of $43(41.8 \%) \quad$ patients. INGN-225 appeared to sensitize SCLC to subsequent chemotherapy. [32].

\section{Discussion And The Future}

The human p53 gene spans $20 \mathrm{~kb}$ chromosome band 17p13.1.It is nuclear phosphoprotein compound having 393 amino acids in human. It regulates gene expression and controls several key genes involved in the growth regulation. The p53 itself is controlled by multiple negative regulators and activators to ensure protection of genome, and thus protecting the organism from cancers. In most of the cancers this p53 pathway is altered by mutation of p53 gene itself or altered sensitivity and effectiveness of p53 pathways. Cancer cells are exceptionally sensitive to reactivation of p53 function and therefore the treatment that achieves this object is most effective therapeutic modality.

Among many genetic lesions, mutational activation of p53 tumour suppressor, "the guardian of Genome" is the most frequently found event in $50 \%$ of human cancers. p53 plays a critical role in tumour suppression, mainly by, inducing growth arrest, apoptosis and senescence as well as blocking angiogenesis. In addition p53 confers to the cancer cell, sensitivity for chemoradiation. Thus p53 becomes most appalling target for mechanism driven anticancer drug discovery. Cancer is a complex disease with multiple genetic and epigenetic alterations. Genetic alterations in any given cancer, even those originating from same tissue/organ, could have dramatic differences. Conversely cancer derived from different tissue/organ may have similar alterations' in a given signalling pathway. Thus an effective personalised cancer therapy requires a thorough understanding of genetic and epigenetic alterations of each individual cancer, followed by rational design of 
combination therapies which targets these altered molecule and pathways. This knowledge can be utilised to target p53 and its regulators in an individual tumour basis, e.g. in wild p53 containing tumours, chemoradiation can be used along with p53 activating or p53 reactivating drugs or drug acting through a synthetic lethal mechanism that could increase the efficacy against tumour with mutant p53. Moreover the synergistic effect on cancer cell killing of p53 drugs plus chemoradiation allows lower dose regimen and thus reduced drug/radiation toxicity to normal tissues.

P53 drugs can be used along with other mechanism driven drugs to activate or synergistic effect by targeting the same signalling pathway both upstream and downstream. e.g. combination of Nutlins or MI-29, which activates p53 but with an adverse effect of Mdm2 accumulation with Smac mimetic drugs, which disrupts XIAP-caspase inhibitory binding to release activated caspase. [33]. A recent report showed that Mdm2 causes the accumulation by XIAP. This mechanism driven combination would lead to apoptosis induced by p53 being fully executed by activated caspase.

As we gain better understanding of p53 signalling pathways, additional p53 related targets, upstream and downstream of p53, can be identified and validated for future discovery of novel compounds that target p53 signalling pathways. [34], [35]

One promising future direction is chemoprevention. It is aimed to kill cancer prone cells at the early stage of carcinogenesis to prevent tumour formation later. Currently our efforts are focussed on cancer therapy with p53 drugs but chemoprevention could be an exciting future development.

\section{References}

[1]. L chen, DM Gilkes, Y Pan, WS Lane, and J Chen. ATM and Chk2- dependent phosphorylation of MDMX contribute to p53 activation after DNA damage. EMBO J. 24, 2005, 3411-3422.

[2]. S Hong, RV Pusapati, JT powers, and DG Jhonson. Oncogene and the DNA damage response: Myc and E2F1 engage the ATM signalling pathway to activate p53 and induce apoptosis. Cell cycle, 5, 2006, 801

[3]. J Nakono, CL Huang, Liu, M Ueno, S Sumitomo, and H Yokomise. Survivin gene expression is negatively regulated by the p53 tumour suppressor gene in non small cell lung cancer. Int j Oncol, 27, 2005, 1215-1221.

[4]. Oren m. Decision making by p53: life, death and cancer. Cell Death Differ, 10, 2003, 431-42.

[5]. Goh AM, Coffill CR, and Lane DP. The role of mutant p53 in human cancer. J Pathol, 223, 2011, 116-26.

[6]. Jung MS, Yun J, and Chae HD, et al. p53 and its homologues, p63 and p73, induce a replicative senescence through inactivation of NF-Y transcription factor. Oncogene, 20, 2001, 5818-25.

[7]. Gohler T, Jager S, and Warnecke G, et al. Mutant p53 proteins bind DNA in a DNA structure-selective mode. Nucleic Acids Res, 33, 2005, 1087-100

[8]. Song H, Hollstein M, and Xu Y. p53 gain-of-function cancer mutants induce genetic instability by inactivating ATM. Nat Cell Biol, 9, 2007, 573-80.

[9]. Roth JA, Nguyen D, and Lawrence DD, et al. Retrovirus mediated wild type p53 gene transfer to tumours of patients with lung cancer. Nat Med, 2, 1996, 985-91.

[10]. Pan JJ, Zhang SW, Chen CB, et al. Effect of recombinant adenovirus-p53 combined with radiotherapy on long term prognosis of advanced nasopharyngeal carcinoma. J Clin Oncol, 27, 2009, 799-804.

[11]. Nemunaitis J, senzer N, sarmiento S, Zhang YA, Arzaga R, and Sands B. A phase I trial of intravenous infusion of ONYX-015 and enbrel in solid tumour patients. Cancer Gene Ther, 14, 2007, 885-93.

[12]. Momand J, Jung D, Wilczynski S, and Niland J. the Mdm2 gene amplification database. Nucleic Acids Res, 26, 1998, 3453-9.

[13]. Vassilev LT, Vu BT, Graves B, et al. In vivo activation of the p53 pathway by small molecule antagonists of Mdm2. Science, 303, 2004, 844-8.

[14]. Issaeva N, Bozko P, and Enge M, et al. Small molecule RITA binds to p53, blocks p53-HDM-2 interaction and activates p53 function in tumors. Nat Med, 10, 2004, 1321-8.

[15]. Shangari S, Qin D, and Mc Eachern D, et al. Temporal activation of p53 by a specific Mdm2 inhibitor is selectively toxic to tumours and leads to complete tumour growth inhibition. Proc Natl Acad Sci. USA, 105, 2008, 3933-8.

[16]. Saha MN, qiu L, and Chang H. Targeting p53 by small molecules in haematological malignancies. J Hematol Oncol, 6, $2013,23$.

[17]. Brachmann RK, Yu K, Eby Y, Pavletich NP, and Boeke JD. Genetic selection of intragenic suppressor mutations that reverse the effect of common p53 cancer mutations. EMBO J. 17 (7), 1998, 1847-1859.

[18]. Selivanova G and Wiman KG. Reactivation of mutant p53: molecular mechanisms and therapeutic potential. Oncogene, 26(15), 2007, 2243-2254.

[19]. Bykov VJ, Issaeva N, Shilov A, Hultcrantz M, Pugacheva E, Chumakov P, Bergman J, Wiman KG and Selivanova G. Restoration of the tumour suppressor functions to mutant p53 by a low molecular weight compound. Nat Med, 8(3), 2002, 282-288.

[20]. Boeckler FM, Joerger AC, and Jaggi G. et al. Targeted rescue of a destabilized mutant of p53 by an in silico screened drug. Proc Natl Acad Sci USA ,105, 2005, 10360-5.

[21]. Peng y, Li c, Chan L, Sebti S, and Chen J. Rescue of mutant p53 transcription function by ellipticine. Oncogene 22(29), 2003, 4478-4487.

[22]. North S, EL-Ghissassi F, Pluquet O, Verhaegh G, and Hainaut P. The cytoprotective aminothiol WR1065 activates p21 waf-1 and down regulates cell cycle progression through a p53-dependent pathway. Oncogene 19 (9), 1206-1214.

[23]. Foster BA, Coffey HA, Morin MJ, and Rastinejad F. Pharmacological rescue of mutant p53 conformation and function. Science, 286, 1999, 2507-10

[24]. Weinmann L, Wischhusen J, Demma MJ, Naumann U, Roth P, Dasmahapatra B, and Weller M. A novel p53 rescue compound induces p53-dependent growth arrest and sensitises glioma cells to Apo2L/TRAIL-induced apoptosis. Cell death Differ 15(4), 2008, 718-729.

[25]. Wang w, Takimoto R, Rastinejad F and EL-Deiry WS. Stabilization of p53 by CP-31398 inhibits ubiquitination without altering phosphorylation at serine 15 or 20 or mdm2 binding. Mol Cell Biol 23(6), 2003, 2171-2181. 
[26]. Demma M, Maxwell E, Ramos R, et al. SCH529074, a small molecule activator of mutant p53, which binds p53 DNA binding domain(DBD), restores growth-suppressive function to mutant p53 and interrupts HDM2-mediated ubiquitination of wild type p53. J Biol Chem, 285, 2010, 10198-212.

[27]. yang A, Kaghad M, wang Y, Gillett E, fleming MD, Dotsch V, Andrews NC, Caput D, and Mckeon F. P63, a p53 homolog at 3q2729, encodes multiple products with transactivating, death inducing, and dominant-negative activities. Mol Cell 2(3), 1998, 305-316.

[28]. Vilgelm A, EL-Rifai W, and Zaika A. Therapeutic prospects for p73 and p63: rising from the shadow of p53. Drug Resist Updat, 11(4-5), 2008, 152-163.

[29]. Li D, Marchenko ND, and Schulz R, et al. Functional inactivation of endogenous MDM2 and CHIP by HSP90 causes aberrant stabilization of mutant p53 in human cancer cells. Mol Cancer Res, 9, 2011, 577-88.

[30]. Li D, Marchenko ND, and Moll UM. SAHA shows preferential cytotoxicity in mutant p53 cancer cells by destabilizing mutant p53 through inhibition of the HDAC6-Hsp90 chaperone axis. Cell Death Differ 18, 2011, 1904-13.

[31]. Speetjens FM, Kuppen PJ, and Welters MJ, et al. Induction of p53-specific immunity by a p53 synthetic lung peptide vaccine in patients treated for metastatic colorectal cancer. Clin Cancer Res, 15, 2009, 1086-95.

[32]. Chiappori AA, soliman H, Janssen WE, Antonia SJ, Gabrilovich DI. INGN-225: a dendritic cell-based p53 vaccine (Ad.p53-DC) in small cell lung cancer: observed association between immune response and enhanced chemotherapy effect. Expert Opin Biol Ther, 10, 2010, 983-91.

[33]. Lu J, Bai L, Sun H, Nikolovaska-coleska Z, McEachern D, Qiu S, Miller RS, Yi H, Shangary S, and Sun Y, et al. SM-164:a novel Bivalent Smac mimetic that induces apoptosis and tumour regression by concurrent removal of the blockade of cIAP-1/2 and XIAP. Cancer Res 68 (22), 2008, 9384-9393.

[34]. Sun Y. P53 and its downstream proteins or molecular targets of cancer. Mol carcinog 45(6), 2006, 409-415.

[35]. Hupp TR, lane DP, and Ball KL. Strategies for manipulating the p53 pathway in the treatment of human cancer. Biochem J 352 (Pt 1), 2000, 1-17. 